\title{
Experimental and numerical investigation of ram extrusion of bread dough
}

\begin{abstract}
An experimental and numerical study on ram extrusion of bread dough was conducted. A laboratory ram extrusion rig was designed and manufactured, where dies with different angles and exit radii were employed. Rate dependent behaviour was observed from tests conducted at different extrusion speeds, and higher extrusion pressure was reported for dies with decreasing exit radius. A finite element simulation of extrusion was performed using the adaptive meshing technique in Abaqus. Simulations using a frictionless contact between the billet and die wall showed that the model underestimates the response at high entry angles. On the other hand, when the coefficient of friction value was set to 0.09 as measured from friction experiments, the dough response was overestimated, i.e. the model extrusion pressure was much higher than the experimentally measured values. When a critical shear stress limit, Ünax, was used, the accuracy of the model predictions improved. The results showed that higher die angles require higher Ünax values for the model and the experiments to agree.
\end{abstract}

Keyword: Adaptive meshing; Bread dough; Critical shear stress limit; Extrusion pressure 